\title{
MPK3/MPK6 are involved in iron deficiency-induced ethylene production in Arabidopsis
}

\author{
Lingxiao Ye ${ }^{1 \dagger}$, Lin $\mathrm{Li}^{1+}$, Lu Wang ${ }^{1,2 \dagger}$, Shoudong Wang ${ }^{1}$, Sen $\mathrm{Li}^{1}$, Juan $\mathrm{Du}^{1}$, Shuqun Zhang ${ }^{1}$ \\ and Huixia Shou ${ }^{1 *}$ \\ ' State Key Laboratory of Plant Physiology and Biochemistry, College of Life Sciences, Zhejiang University, Hangzhou, China, \\ ${ }^{2}$ Key Laboratory of Tea Biology and Resources Utilization, Ministry of Agriculture, National Center for Tea Plant Improvement, \\ Tea Research Institute, Chinese Academy of Agricultural Sciences, Hangzhou, China
}

Iron $(\mathrm{Fe})$ is an essential micronutrient that participates in various biological processes important for plant growth. Ethylene production induced by Fe deficiency plays important roles in plant tolerance to stress induced by Fe deficiency. However, the activation and regulatory mechanisms of 1-Aminocyclopropane-1-carboxylic acid synthase (ACS) genes in this response are not clear. In this study, we demonstrated that Fe deficiency increased the abundance of ACS2, ACS6, ACS7, and ACS11 transcripts in both leaves and roots as well as the abundance of ACS8 transcripts in leaves and ACS9 transcripts in roots. Furthermore, we investigated the role of mitogen-activated protein kinase 3 and 6 (MPK3/MPK6)-regulated ACS2/6 activation in Fe deficiency-induced ethylene production. Our results showed that MPK3/MPK6 transcript abundance and MPK3/MPK6 phosphorylation are elevated under conditions of Fe deficiency. Furthermore, mpk3 and mpk6 mutants show a lesser induction of ethylene production under Fe deficiency and a greater sensitivity to Fe deficiency. Finally, in mpk3, mpk6, and acs2 mutants under conditions of Fe deficiency, induction of transcript expression of the Fe-deficiency response genes FRO2, IRT1, and FIT is partially compromised. Taken together, our results suggest that the MPK3/MPK6 and ACS2 are part of the Fe starvation-induced ethylene production signaling pathway.

\footnotetext{
Keywords: Arabidopsis, ethylene, Fe deficiency, mitogen-activated protein kinase (MPK), 1-Aminocyclopropane1-carboxylic acid synthase (ACS)
}

\section{INTRODUCTION}

Iron $(\mathrm{Fe})$ is an essential micronutrient that plays an important role in plant growth. It participates in several metabolic processes including respiration, photosynthesis, and chlorophyll biosynthesis (Kobayashi and Nishizawa, 2012). Since Fe is poorly soluble in neutral or basic soils, it is not readily available to plants in these conditions (Kim and Guerinot, 2007). To counteract Fe deficiency, plants have developed a set of responses to control the uptake, utilization, and storage of Fe. Most plants, with the exception of those in the Graminaceae family, use strategy I, which is also known as the reduction strategy. Strategy I plants, via $\mathrm{H}^{+}$-ATPases, excrete protons into the rhizosphere to increase the solubility of Fe. Ferric-chelate reductase oxidases present on the root surface then reduce $\mathrm{Fe}^{3+}$ into $\mathrm{Fe}^{2+}$, after which $\mathrm{Fe}^{2+}$ transporters take $\mathrm{Fe}^{2+}$ into the plant. Consistent with the roles of these proteins in strategy I plants, in Arabidopsis, expression of the 
plasma membrane $\mathrm{H}^{+}$ATPase (AHA2), the major ferric-chelate reductase oxidase (FRO2), and the major $\mathrm{Fe}^{2+}$ transporter (IRT1) is strongly induced under Fe deficiency (Eide et al., 1996; Robinson et al., 1999; Santi and Schmidt, 2009). The Graminaceae family plants use strategy II, which is also known as the chelate strategy (Kim and Guerinot, 2007; Walker and Connolly, 2008). Strategy II plants release phytosiderophores, which can directly bind $\mathrm{Fe}^{3+}$ (Conte and Walker, 2011), into the rhizosphere. The chelated complexes are then transported into the roots through the YS/YSL family of transporters (Curie et al., 2001, 2009).

Recent studies have shown that in strategy I plants, phytohormones such as ethylene, auxin, cytokinins, and nitric oxide (NO) are involved in the regulation of Fe deficiency responses (Romera et al., 2011; Ivanov et al., 2012). In particular, Fe deficiency increases production of ethylene in roots of strategy I plants (Romera et al., 1999; Romera and Alcantara, 2004). It is thought that ethylene regulates $F R O 2$ and IRT1 gene expression through the modulation of the major transcription factor FER or FER-like (Lucena et al., 2006). In support of this, in conditions of Fe deficiency, expression of Arabidopsis FIT, which is homolog of tomato FER decreases upon inhibition of ethylene synthesis or activity, and increases upon addition of ethylene precursor (García et al., 2010). And the expression of Fe-related genes and ferric reductase activity were also induced by ethylene level (Romera and Alcantara, 1994; Li and Li, 2004; Lucena et al., 2006; Waters et al., 2007; García et al., 2010). The genes involved in ethylene biosynthesis and signaling could also be up-regulated under Fe deficiency (García et al., 2010). Ethylene biosynthesis involves three enzymatic steps: (1) S-AdoMet synthetase converts methionine to S-adenosyl-L-methionine (S-AdoMet); (2) SAdoMet is converted to ACC by ACC synthase (ACS); (3) ACC is oxidized by ACC oxidase (ACO) and is thereby converted to ethylene (Yang and Hoffman, 1984; Sato and Theologis, 1989; Zarembinski and Theologis, 1994; Wang et al., 2002; Chae and Kieber, 2005). Unlike ACO, ACS has very low basal activity and can be rapidly increased under conditions that promote ethylene production (Yang and Hoffman, 1984). Thus, ACS is considered to be the rate-limiting enzyme in ethylene biosynthesis.

Arabidopsis has nine genes encoding ACS isoforms that are classified into three types according to the phosphorylation sites in their C-termini. ACS1, ACS2, and ACS6 are the type I ACS isoforms and have phosphorylation sites for mitogen-activated protein kinases (MAPKs) and calcium-dependent protein kinases (CDPKs; Liu and Zhang, 2004; Kamiyoshihara et al., 2010). ACS2 and ACS6 could be regulated by the MAPKs MPK3, and MPK6 at both the transcriptional and posttranslational levels (Liu and Zhang, 2004; Han et al., 2010; Li et al., 2012). Type II ACSs include ACS4, ACS5, ACS8, and ACS9, and have putative CDPK phosphorylation sites, but not MAPK phosphorylation sites, in their C-termini. ACS7 and ACS11 are classified into Type III ACS isoforms, which lack both types of phosphorylation sites. Previous studies have shown that expression of ACS isoforms is tissue-specific, and that different ACS isoforms respond differently to extracellular stimuli (Zarembinski and Theologis, 1994; Wang et al., 2002).
In plants, MAPK cascades, which consist of MAPKKK, MAPKK, and MAPK, play vital roles in development and in a number of stress responses, including those to wounding, pathogen infection, temperature, salinity, drought, osmolarity, ozone, UV irradiation, ROS, and nutrient deficiency (Group et al., 2002; Pedley and Martin, 2005; Zhang et al., 2007; Pitzschke et al., 2009; Rodriguez et al., 2010; Tena et al., 2011). MPK3 and MPK6 can be regulated by different MAPKKs under different stress conditions (Teige et al., 2004; Liu et al., 2007; Takahashi et al., 2007; Wang et al., 2007, 2008, 2010; Xu et al., 2008; Yoo et al., 2008; Zhou et al., 2009). In addition, recent work shows that the MKK9-MPK3/MPK6 cascade is involved in phosphate (Pi) acquisition (Lei et al., 2014). However, the role of MAPKs in regulation of plant responses to Fe deficiency has not been studied.

Using quantitative RT-PCR (qRT-PCR) analysis, we found that the expression of ACS2, ACS6, ACS7, and ACS11 transcripts in both leaves and roots, ACS8 transcripts in leaves and ACS9 in roots were up-regulated by Fe deficiency. Further analysis showed that MPK3/MPK6 participates in Fe deficiencyinduced ethylene production. Loss function in MPK3 and MPK6 suppressed the expression of ACS2, ACS6, and the Fe deficient responses. As a result, the $m p k 3$ and $m p k 6$ plants had a reduced soluble Fe content and severe chlorosis symptoms compared to the wild-type (WT) plants when grown under Fe deficient conditions.

\section{MATERIALS AND METHODS}

\section{Plant Materials}

Arabidopsis thaliana Columbia (Col-0) ecotype was used as the WT control. T-DNA insertion mutant alleles of MPK3 (At3g45640), MPK6 (At2g43790), ACS2 (At1g01480), ACS6 (At4g11280) were described previously (Liu and Zhang, 2004; Wang et al., 2007; Han et al., 2010). The high-order acs mutants generated in Dr. Athanasios Theologis' laboratory (Tsuchisaka et al., 2009) were obtained from the Arabidopsis Biological Resource Center (ABRC). The stock numbers of the high-order ACS mutants acs2/acs4/acs5//acs6/acs7/acs9 and acs1/acs2/acs4/acs5/acs6/acs7/ acs9/acs11 are CS16649 and CS16651, respectively.

\section{Growth Conditions and Treatments}

For hydroponic experiments, seeds were vernalized at $4^{\circ} \mathrm{C}$ for 3 days in the distilled water. Then they were sown in $1.5 \mathrm{ml}$ bottom-cut centrifuge tubes containing $400 \mu \mathrm{L}$ of $0.6 \%$ agarose gel. The tubes were held in the holes of a thin polyurethane raft floating on nutrient solution (Lucena et al., 2006). This arrangement allowed the plants growing in the float to uptake the nutrient solution via the agarose gel. The nutrient solution (without $\mathrm{Fe}$ ) had the following composition: $2000 \mu \mathrm{M} \mathrm{Ca}\left(\mathrm{NO}_{3}\right)_{2}$, $500 \mu \mathrm{M} \mathrm{KH}_{2} \mathrm{PO}_{4}, 750 \mu \mathrm{M} \mathrm{K}_{2} \mathrm{SO}_{4}, 650 \mu \mathrm{M} \mathrm{MgSO} 4,50 \mu \mathrm{M} \mathrm{KCl}$, $1 \mu \mathrm{M} \mathrm{MnSO}, 0.5 \mu \mathrm{M} \mathrm{ZnSO}_{4}, 0.5 \mu \mathrm{M} \mathrm{CuSO}_{4}, 10 \mu \mathrm{M} \mathrm{H}_{3} \mathrm{BO}_{3}$, and $0.05 \mu \mathrm{M}\left(\mathrm{NH}_{4}\right)_{6} \mathrm{Mo}_{7} \mathrm{O}_{24}$. Fe-EDTA was added or not added to the nutrient solution depending on the experiments. The $\mathrm{pH}$ of the nutrient solution was adjusted to 6.0. The growth chamber of seedlings was set $22^{\circ} \mathrm{C}$ day $/ 20^{\circ} \mathrm{C}$ night temperatures, relative 
humidity $60 \%$, and a $10 \mathrm{~h}$ photoperiod at a photosynthetic irradiance of $300 \mu \mathrm{molm}^{-2} \mathrm{~s}^{-1}$ (Lucena et al., 2006).

Experiments using 10 day old seedlings were performed at swimming medium culture as described (Li et al., 2012). After being vernalized at $4^{\circ} \mathrm{C}$ for 3 days, the surface sterilized seeds were sown in liquid half-strength (1/2) Murashige and Skoog (MS) medium and grown in a growth chamber at $22^{\circ} \mathrm{C}$ with continuous $\operatorname{light}\left(70 \mu \mathrm{E} / \mathrm{m}^{-2} \mathrm{sec}^{-1}\right)$. Five-day-old seedlings were transferred to $20-\mathrm{ml}$ gas chromatography (GC) vials with $6 \mathrm{ml}$ of liquid $1 / 2$ MS medium (10 seedlings per vial) and the growth conditions maintained the same as before. Seedlings were grown in $1 / 2$ MS swimming medium for 10 days, and then transferred to $1 / 2 \mathrm{MS}$ medium with or without Fe. Ethylene were measured at 4 day or the indicated day (in the time course analysis) after the treatment, while analysis of transcript abundance was performed at 7 days after treatment.

\section{RNA Isolation and qRT-PCR}

According to the manufacturer's instructions (Invitrogen, Carlsbad, CA, USA), Triozol reagent were used for total RNA isolation. To remove the residual genomic DNA, five micrograms of RNA were then treated with RNase-free DNase I (Takara Bio, Tokyo, Japan). Using M-MLV reverse transcriptase, First-strand cDNA was synthesized (Promega, Madison, WI, USA). qRT-PCR was performed as described previously (Zheng et al., 2009). A LightCycler 480 machine was used for PCR amplification of cDNA (Roche Diagnostics, Basel, Switzerland) with SYBR Premix Ex Taq Kit (Takara Bio). Quantitative assays were performed in triplicate on each sample and the reference gene $\beta$-tubulin was used as an internal control. Transcript levels relative to $\beta$-tubulin were calculated using the formula $2^{-\Delta \Delta \mathrm{Ct}}$. All primer sequences used for the PCR reactions are provided in Supplementary Table 1.

\section{Protein Extraction and Immunoprecipitation Kinase Assay}

Protein extraction was performed as described previously (Ren et al., 2002). Total protein was extracted from whole seedlings by grinding in extraction buffer containing $100 \mathrm{mM}$ HEPES, pH 7.5, $5 \mathrm{mM}$ EDTA, $5 \mathrm{mM}$ EGTA, $10 \mathrm{mM} \mathrm{Na} \mathrm{VO}_{4}, 10 \mathrm{mM}$ $\mathrm{NaF}, 50 \mathrm{mM} \beta$-glycerophosphate, $10 \mathrm{mM}$ dithiothreitol, $1 \mathrm{mM}$ phenylmethylsulfonyl fluoride, $5 \mathrm{~g} \mathrm{ml}^{-1}$ leupeptin, $5 \mathrm{~g} \mathrm{ml}^{-1}$ aprotinin, and 5\% glycerol. Supernatants were transferred into $1.5 \mathrm{~mL}$ tubes after centrifugation at $18,000 \times \mathrm{g}$ for $40 \mathrm{~min}$. Samples should be quickly frozen in liquid nitrogen and stored at $-80^{\circ} \mathrm{C}$ until further analyses. The concentration of protein extracts was determined using the Bio-Rad protein assay kit (BioRad) with bovine serum albumin as a standard (Ren et al., 2002). Twelve micrograms of protein was loaded into each lane and separated by SDS-PAGE.

Immunoprecipitation kinase assay was performed as described (Lee and Ellis, 2007; Tsuda et al., 2009). Antip44/42 MAPK (Erk1/2) (Thr202/Tyr204) antibody, which specifically recognizes the dually phosphorylated-pTXpY- motif in phospho-MPK3 and phospho-MPK6, was used to detecting the amount of phosphorylated MPK3 and MPK6, i.e., the activities of the MPK3 and MPK6. The secondary antibody was a horseradish peroxidase-conjugated goat anti-rabbit IgG antibody. The protein membranes were visualized with an Enhanced Chemiluminescence Kit (Roche) and then it was exposed to X-ray film.

\section{Ethylene Measurement}

After treatment, the GC vials which contain Arabidopsis seedlings were flushed and capped immediately. Twenty-four hours before the measurement of the ethylene production, the GC vials were flushed with fresh air to remove the ethylene accumulated before the day. Ethylene accumulated in the headspace of the GC vials over a $24 \mathrm{~h}$ period were determined by gas chromatography and mass spectrometry at indicated times (Kim et al., 2003; Liu and Zhang, 2004). Then the seedlings were harvested and weighed. Samples were frozen in liquid nitrogen for future analysis.

\section{Chlorophyll Content Analysis}

The SPAD value (a measure of total chlorophyll content) of the fully expanded youngest leaves was measured by a portable chlorophyll meter (SPAD-502; Konica Minolta Sensing, JP).

\section{Measurement of Soluble Fe Concentration}

To determine the concentration of soluble $\mathrm{Fe}$ in plants, approximately $0.5-1 \mathrm{~g}$ of new leaves of treated seedlings were ground in liquid nitrogen. Five volumes of deionized water were added to extract the soluble $\mathrm{Fe}$ at room temperature. After centrifugation, the supernatant was collected in new tubes (Zheng et al., 2009). Inductively coupled plasma mass spectrometry (ICP-MS, Agilent 7500ce, Santa Clara, CA, USA) was used for Fe concentration measurement.

\section{RESULT}

\section{Effect of Fe Deficiency on Ethylene Production}

A previous study found that ethylene production in the roots of the strategy I plants pea, tomato, and cucumber increased under conditions of $\mathrm{Fe}$ deficiency (Romera et al., 1999). A time-course experiment was carried out with WT seedlings to investigate whether Fe deficiency induced ethylene production in Arabidopsis (Figure 1). To accurately measure the ethylene production at different treatment time, the GC vials were flushed with fresh air at $24 \mathrm{~h}$ prior to the measurement. Ethylene accumulated over a $24 \mathrm{~h}$ period was determined at the day indicated. Upon initiation of Fe deprivation, the induction of ethylene production began at the 2 nd day and reached its maximum level at the 4th day. The maximum level of ethylene production in $\mathrm{Fe}$ deficient conditions was approximately three times higher than that in Fe sufficient conditions. After reaching its maximum level, ethylene levels decreased gradually and dropped to basal levels at 5 days after initiation of Fe deficient conditions. 


\section{Effect of Fe Deficiency on ACS Transcription}

Using semi-quantitative PCR, a previous study demonstrated that several ACS genes, including ACS4, ACS6, and ACS9, are

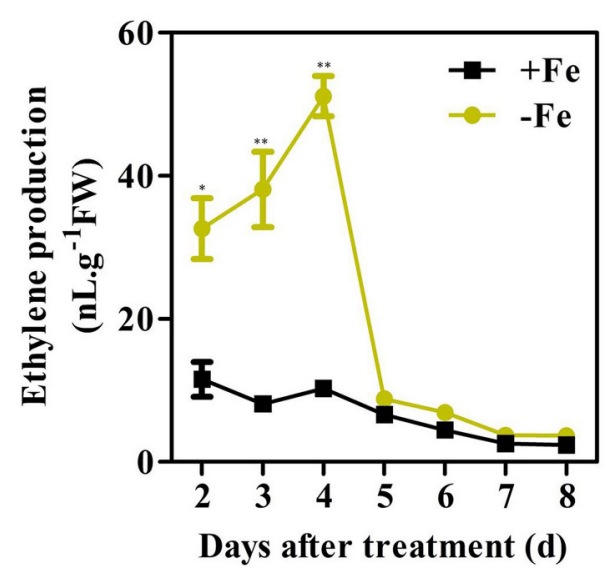

FIGURE 1 | Time-course analysis of ethylene production in WT plants under Fe-sufficient (+Fe) and -deficient (-Fe) conditions. Ten-day-old seedlings were transferred to Fe-sufficient (50 $\mu \mathrm{M}$ EDTA-Fe) or Fe-deficient (0 $\mu \mathrm{M}$ EDTA-Fe) nutrient solutions. For each time point shown, ethylene accumulated over a $24 \mathrm{~h}$ period from 10 seedlings in the headspace of GC vials was measured. Data are shown as the mean $\pm \operatorname{SEM}(n=3)$. Columns marked with "*” indicate a significant difference $(P<0.05)$, and "**” indicate a highly significantly difference $(P<0.01)$. up-regulated under the stressful conditions of Fe deficiency (García et al., 2010). To identify all the ACS isoforms involved in ethylene production under Fe deficiency, we used qRT-PCR to measure the expression of all nine ACS genes in Arabidopsis grown under Fe-sufficient and Fe-deficient conditions. We were able to detect the expression of eight of the nine ACS genes (all but $A C S 1$ ) in leaf, root, or both (Figure 2). The transcript abundance of ACS2, ACS6, ACS7, and ACS11 increased significantly in both leaf and root after 7 days of Fe deprivation. ACS 8 transcripts were detected only in leaf, and were four-fold more abundant under $\mathrm{Fe}$ deficient conditions than under Fe sufficient conditions. While ACS4 transcripts were also detected only in leaf, Fe deficiency did not lead to a significant change in ACS4 mRNA. ACS5 mRNA and ACS9 mRNA were detected only in root. ACS9 expression was over 30-fold higher in conditions of Fe deficiency compared to those of Fe sufficiency, whereas expression of ACS5 was reduced. These results suggest that ACS2, ACS6, ACS7, ACS8, ACS9, and ACS11 may contribute to ethylene induction under conditions of Fe deficiency.

\section{Mutation of ACS2 Suppressed Ethylene Production Induced by Fe Deficiency}

As the rate-limiting enzyme in ethylene biosynthesis, ACS is well positioned to influence ethylene production. To explore the involvement of ACS isoforms in Fe deficiency-induced ethylene production, acs2, acs6, and high-order acs mutants were used. In these mutants, expression of corresponding ACS genes was abolished (Supplementary Figure 1A). Under
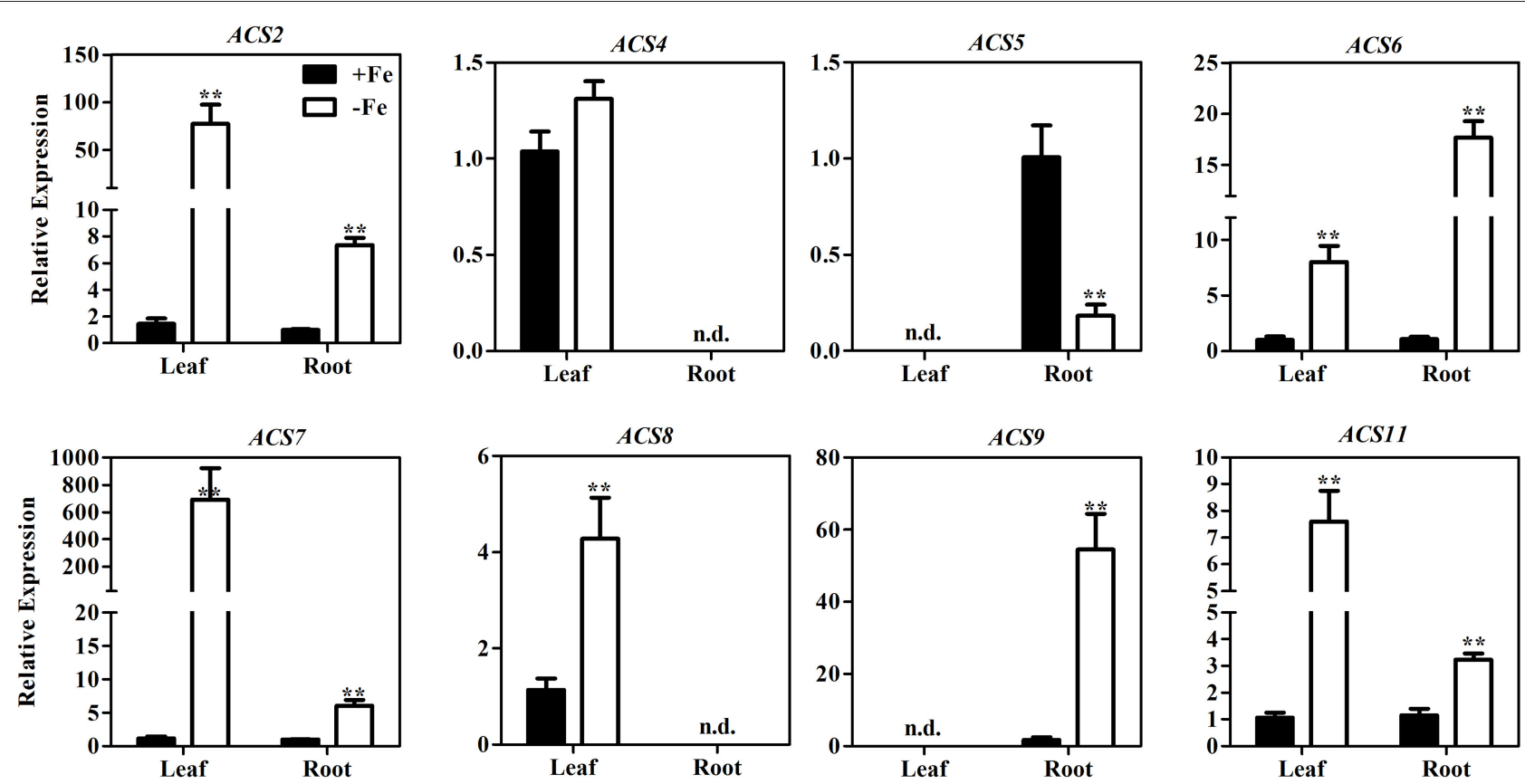

FIGURE 2 | Transcription levels of ACS genes in the leaves and roots of WT seedlings under Fe-sufficient (+Fe) and -deficient (-Fe) conditions. One-month-old seedlings were transferred to +Fe (50 $\mu \mathrm{M}$ EDTA-Fe) or -Fe (0 $\mu \mathrm{M}$ EDTA-Fe) nutrient solutions for 7 days. Leaves and roots were collected for RNA extraction. All values are expressed relative to the expression level under Fe-sufficient conditions (control-set to 1.0) as appropriate. Data are shown as the mean $\pm \operatorname{SEM}(n=3)$. Column marked with "**" indicate a highly significantly difference $(P<0.01)$. n.d. indicates "not detectable." 
conditions of Fe deficiency, acs 2 seedlings produced ethylene at a level that was only $60 \%$ of that in WT seedlings (Figure 3). Surprisingly, ACS6 gene mutation did not affect Fe-induced ethylene induction, as acs 6 seedlings produced the same amount of ethylene as the WT. Ethylene production in high-order acs mutants (acs2/acs4/acs5/acs6/acs7/acs9 and acs1/acs2/acs4/acs5/ acs6/acs7/acs9/acs11) was very low in both $\mathrm{Fe}$ sufficient and $\mathrm{Fe}$ deficient conditions.

\section{Mutation of ACS2 Suppressed the Upregulation of Fe Deficiency-responsive Genes}

Ethylene regulates expression of FER (or FER-like) and thereby regulates gene expression of downstream Fe transporter, ferric reductase, and $\mathrm{H}^{+}$-ATPase (Lucena et al., 2006). We did

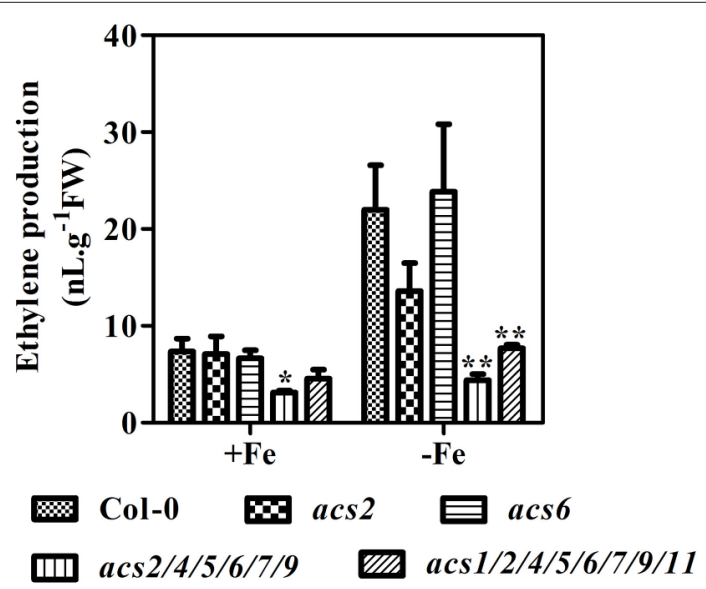

FIGURE 3 | Ethylene production of WT, acs mutant plants under Fe-sufficient (+Fe) and -deficient ( $-\mathrm{Fe})$ conditions. Ten-day-old seedlings were transferred to Fe-sufficient ( $50 \mu \mathrm{M}$ EDTA-Fe) or -deficient ( $0 \mu \mathrm{M}$ EDTA-Fe) nutrient solutions. Ethylene accumulated over a $24 \mathrm{~h}$ period from 10 seedlings in the headspace of GC vials was measured at 4 day after the treatment. Data are shown as the mean $\pm \operatorname{SEM}(n=3)$. Columns marked with "*" indicate a significant difference $(P<0.05)$, and "**” indicate a highly significantly difference $(P<0.01)$. not observe obvious phenotypic differences between WT and acs 2 or acs 6 mutants in $\mathrm{Fe}$ sufficient or deficient conditions (Supplementary Figure 2A). However, in conditions of $\mathrm{Fe}$ deficiency, upregulation of Fe deficiency-responsive genes was attenuated in acs 2 mutants (Figure 4) and the high order ACS mutant (Supplementary Figure 3 Specifically, expression levels of FIT, FRO2, and IRT1 genes in acs 2 mutants were reduced 40, 50, and 33\% from levels in WT seedlings, respectively. In contrast, the induction of Fe deficiency-responsive gene expression did not change in the acs 6 mutants. To confirm the reduction of FRO2 expression indeed affected the Ferric-chelate reductase (FCR) activity, FCR assay was performed on acs2, acs6, and high order ACS mutants. Compared to the WT plants, the FCR activity in acs 2 was slightly reduced, but not significant (Supplementary Figure 2B). In contrast, FCR activity in the high order ACS mutants were significantly reduced. The FCR activity is in agreement with the FRO2 transcript level.

\section{Fe Deficiency Activates MPK3/MPK6 at Both Transcript and Protein Levels}

Previous research has shown that MPK3/MPK6-mediated phosphorylation of ACS2 and ACS6 proteins leads to their stabilization and accumulation (Liu and Zhang, 2004; Han et al., 2010; Li et al., 2012). qRT-PCR results confirmed that ACS2 and ACS6 transcript levels are increased under Fe deficiency conditions (Figure 2). To further understand the roles of MPK3 and MPK6 in Fe deficiency-induced ethylene production, both transcript and enzymatic activity levels of MPK3 and MPK6 were determined. Results showed that levels of MPK3 and MPK6 transcripts were significantly increased under $\mathrm{Fe}$ deficiency (Figure 5A). Specifically, expression of MPK3 was induced eight-fold in leaf and 2.5-fold in root, whereas that of MPK6 was induced only 1.8-fold in leaf and 2.8-fold in root. Furthermore, immunoprecipitation kinase assay was performed using anti-p44/42 MAPK (Erk1/2) (Thr202/Tyr204) antibody to detect the amount of phosphorylated MPK3 and MPK6, (Figure 5B). While the activation of MPK6 persisted 4-5 days after treatment, the activation of MPK3 persisted only 1-2 days after treatment. Taken together, these results indicate that the
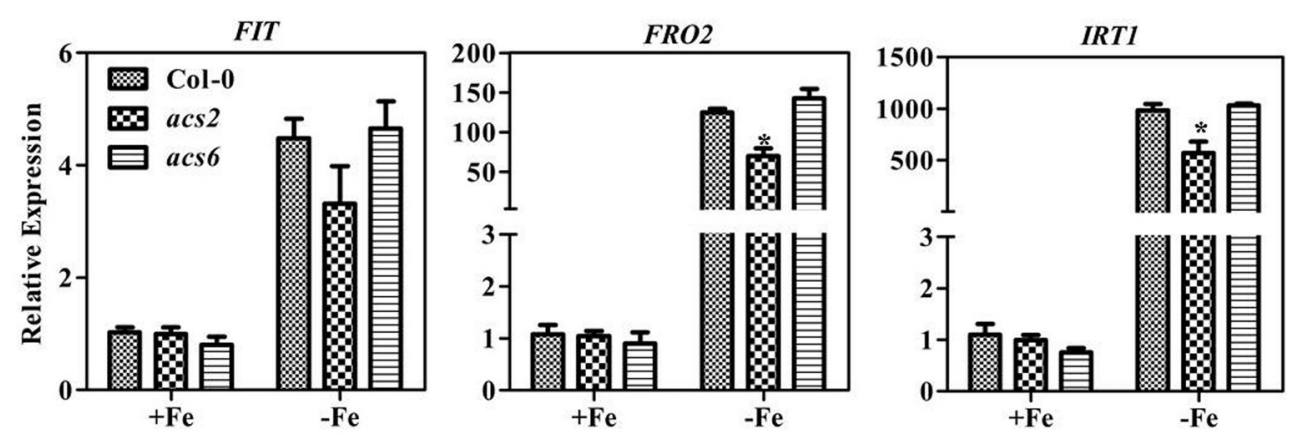

FIGURE 4 | Transcription levels of Fe-deficiency responsive genes in acs2 and acs6 mutant plants. Ten day old seedlings cultured in swimming medium were transferred to Fe-deficient medium for 7 days and sampled for RNA extraction. All values are expressed relative to the expression level under Fe-sufficient conditions (control-set to 1.0) as appropriate. Data are shown as the mean \pm SEM $(n=3)$. Column marked with "*” indicate a significant difference $(P<0.05)$. 

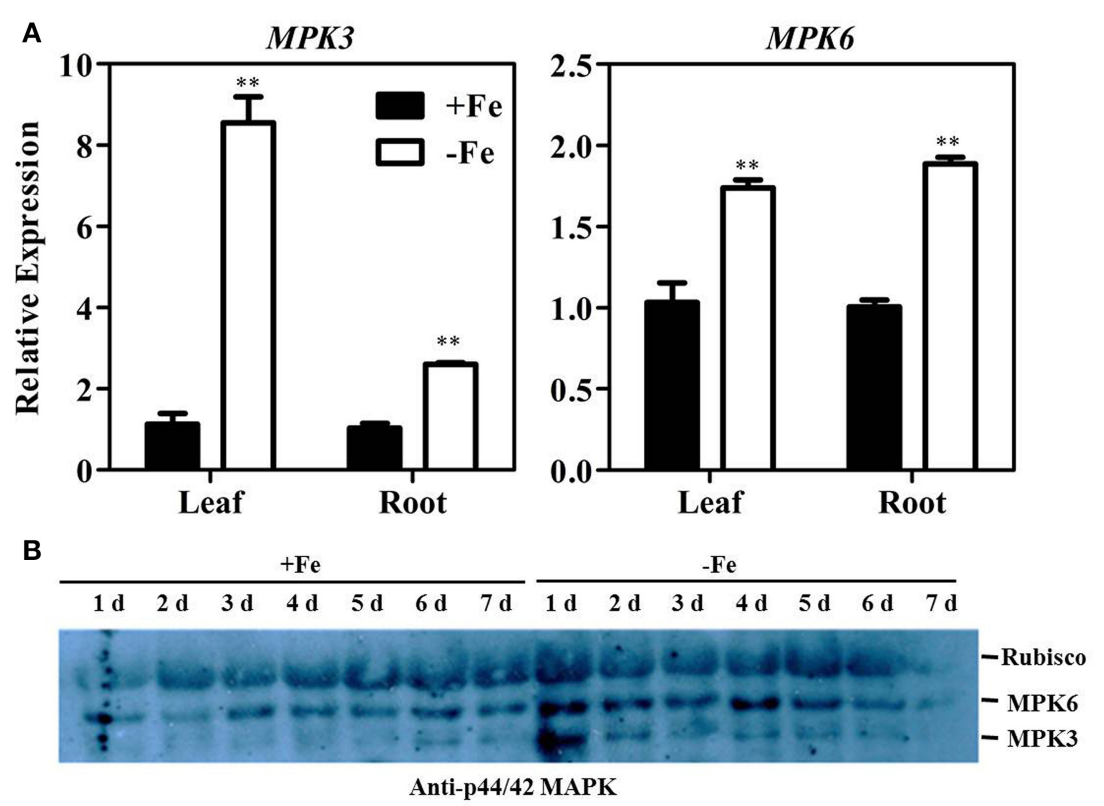

FIGURE 5 | Fe deficiency activates MPK3 and MPK6. (A) Transcription levels of MPK3 and MPK6 in the leaf and root of WT seedlings under Fe-sufficient (50 $\mu$ M EDTA-Fe) and-deficient (0 $\mu \mathrm{M}$ EDTA-Fe) conditions. All values are expressed relative to the expression level under Fe-sufficient conditions (control-set to 1.0) as appropriate. Data are shown as the mean \pm SEM $(n=3)$. (B) Immunoprecipitation kinase assay of MPK3 and MPK6 activity using Anti-p44/42 MAPK antibody. Ten-day-old seedlings were transferred to Fe-sufficient or -deficient nutrient solutions. Column marked with "**” indicate a highly significantly difference $(P<0.01)$.

transcript abundance and enzymatic activities of MPK3 and MPK6 were induced by Fe deficiency.

\section{Loss of MPK3 or MPK6 Resulted in Reduction of Ethylene Production and the Expression Level of ACS2 and ACS6 Under Fe Deficiency}

To further demonstrate that the MPK3/MPK6 cascade participates in Fe-induced ethylene production, two $m p k$ mutants for each MPK were used in subsequent experiments: $m p k 3-1, \quad m p k 3-2, \quad m p k 6-2$, and mpk6-3. Phosphorylation enzymatic activity assay showed that the mutants lost the function of the corresponding kinase activity (Supplementary Figure 1B). Fe deficiency-induced ethylene production in $m p k 3-1$ and $m p k 3-2$ was reduced by 15 and 28\%, respectively, compared to that of WT seedlings (Figure 6A). In $m p k 6$ mutants, reduction of $\mathrm{Fe}$ deficiency-induced ethylene biosynthesis was much more severe. Compared to WT seedlings, both mpk6 mutants maintained only $50 \%$ of ethylene production. These results provide further support for the involvement of MPK3 and MPK6 in ethylene production under Fe deficiency.

ACS2 and ACS6 are known to be regulated by the MPK3/MPK6 at both transcriptional level and posttranslational level (Li et al., 2012). To determine whether mutation in MPK3 and MPK6 would affect the expression ACS2 and ACS6, qRTPCR was performed in the mpk3 and mpk6 plants grown under Fe sufficient or deficient conditions. While Fe deficiency induced the expression of ACS2 and ACS6, the up-regulation was suppressed in the $m p k 3$ and $m p k 6$ mutants (Figure 6B).

\section{The MPK3 and MPK6 Mutants Showed Severe Chlorosis Under Fe Deficiency}

To further investigate the role of MPKs in Fe deficiency, the growth performance and the expression of the Fe deficiencyresponsive genes were investigated in the $m p k$ mutants. Under Fe sufficient conditions, growth performance of WT seedlings was not significantly different from that of mutants (Figures 7A,B). However, under Fe deficient conditions, the mutants, especially the mpk3-1 and mpk6-3 mutants, were smaller and showed more severe chlorosis than WT seedlings (Figures 7A,B). Consistent with the mutants' chlorosis phenotypes, chlorophyll content, measured as the leaf SPAD value, was lower in mpk3-1, mpk6-2, and mpk6-3 mutants than in WT seedlings (Figure 7C).

To determine whether the severe chlorosis phenotype in the $m p k 3$ and mpk6 mutants was due to a decrease in leaf $\mathrm{Fe}$ content, the total leaf $\mathrm{Fe}$ concentrations in the mutants were measured. Fe concentrations were at the same level in WT seedlings and mutants (Supplementary Figure 4). To further investigate the relationship between Fe content and SPAD value, soluble $\mathrm{Fe}$ concentrations in the leaves of mutants and WT seedlings were determined. Our results showed that soluble Fe in mpk3-2 and both mpk6 mutants was marginally lower than that in the WT (Figure 7D). Furthermore, Fe deficiency-responsive gene expression was suppressed in both the mpk3-2 and mpk62 mutants compared to WT seedlings (Figure 8). Specifically, 

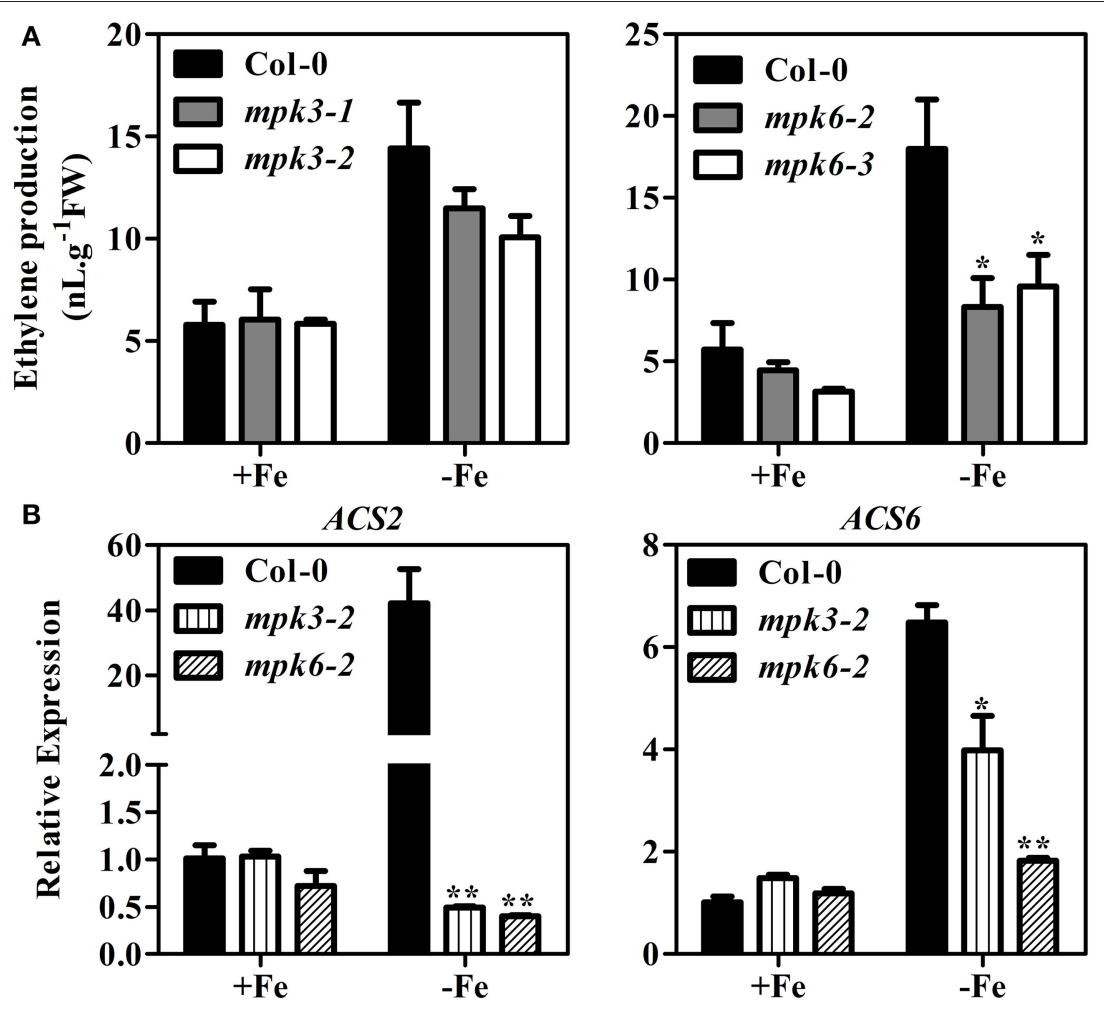

FIGURE 6 | Ethylene production and expression of $\mathbf{A C S} 2$ and $\mathbf{A C S} 6$ in $\boldsymbol{m p k} \mathbf{3}$ and $\boldsymbol{m p k} \mathbf{6}$ mutants. (A) Ethylene production in mpk3 and mpk6 mutants. (B) Transcript abundances of ACS2 and ACS6 genes in mpk3 and mpk6 mutants. Ten-day-old seedlings were transferred to Fe-sufficient (50 $\mu$ M EDTA-Fe) or -deficient $(0 \mu \mathrm{M}$ EDTA-Fe) nutrient solutions. Ethylene accumulated over a $24 \mathrm{~h}$ period from 10 seedlings in the headspace of GC vials was measured at 4 days after the treatment. qRT-PCR was conducted using RNA extracted from whole seedlings at 7 days after - Fe treatment. Data are shown as the mean \pm SEM ( $n=3$ ). Columns marked with "*” indicate a significant difference $(P<0.05)$, and "**” indicate a highly significantly difference $(P<0.01)$.

the induction of FRO2 and IRT1 in both mutants dropped to nearly $50 \%$ of WT levels. In the mpk3-2 mutant, expression of FIT dropped to $30 \%$ of that in WT seedlings.

\section{DISCUSSION}

In strategy I plants, ethylene production is induced by $\mathrm{Fe}$ deficiency stress (Romera et al., 1999; Romera and Alcantara, 2004), which in return, functions as a positive regulator of Fe deficiency response (Romera and Alcantara, 1994; Li and Li, 2004; Lucena et al., 2006; Waters et al., 2007; García et al., 2010; Lingam et al., 2011; Romera et al., 2011). The aim of the study was to explore the regulatory mechanism of this physiological response. In the report, we demonstrated the involvement of MPK3 and MPK6 in the Fe-deficient induced ethylene production based on the following evidence. Firstly, the transcript abundance and enzymatic activities of MPK3 and MPK6 increased under Fe deprivation condition (Figure 5). ACS2 and ACS6 are known to be regulated by the MPK3/MPK6 at both transcriptional level mediated by a transcription factor and posttranslational level via the direct protein phosphorylation by MPK3/MPK6 (Li et al., 2012). The up-regulated expression of ACS2, ACS6 in Fe-deficient plants at both root and leaf tissues (Figure 6B) is likely a consequence of up-regulation of MPK3 and MPK6. Secondly, the Fe-deficient induced ethylene production decreased in the $m p k 3$ and $m p k 6$ mutants (Figure 6A). Thirdly, the expression of Fe acquisition genes, FIT, FRO2, and IRT1 in mpk3 and mpk6 mutants was less up-regulated by $\mathrm{Fe}$ deprivation than that in the WT (Figure 8). As a result, the $m p k 3$ and $m p k 6$ plants had a reduced soluble Fe content and severe chlorosis symptoms compared to the WT plants when grown under Fe deficient conditions (Figure 7).

The Fe-deficient induced ethylene production was not completely abolished in mpk3, mpk6, acs 2 , and acs 6 mutants (Figure 3, Figure 6). It suggests that other ACS isoforms are also involved in the process. Indeed, other than ACS2 and ACS6, the expression of ACS7, ACS9, and ACS11 genes in roots, and $A C S 7, A C S 8$, and $A C S 11$ in leaves were also up-regulated by Fe deficiency (Figure 1). Changes in the expression of the above ACS isoforms should also contribute to the Fe-deficient induced ethylene production. García et al. (2010) examined the expression of ACS4, ACS6, ACS9, and ACS11 in Arabidopsis roots in response to Fe deficiency. They found that the expression of ACS4, ACS6, and AC9 were up-regulated by Fe-deficiency. In contrast to that, the expression of ACS4, which was upregulated by Fe-deficiency in that research (García et al., 2010), was not detected in the study. In addition, the increased expression of 

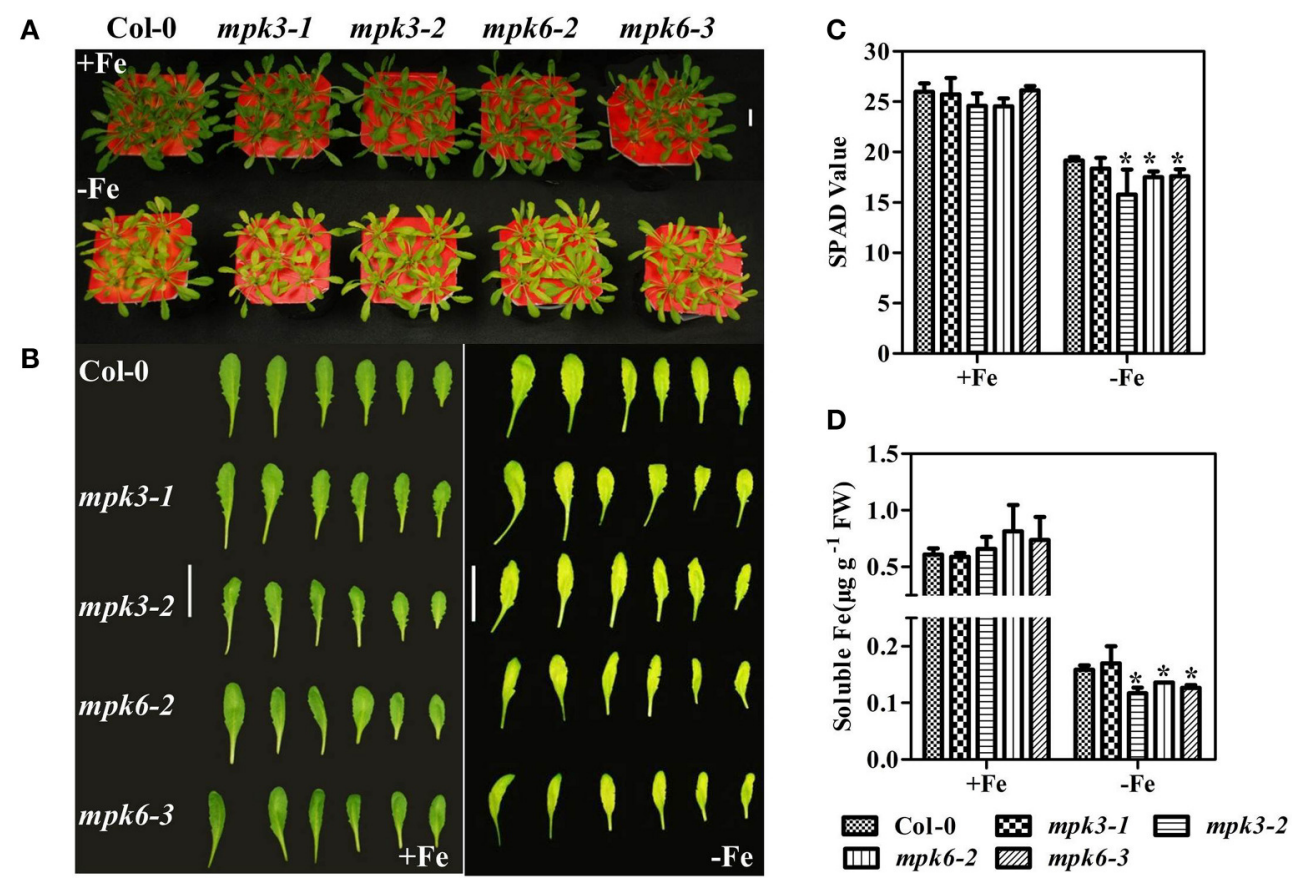

FIGURE 7 | Phenotypes of $\boldsymbol{m p k 3}$ and $\boldsymbol{m p k 6}$ mutants grown in Fe-sufficient or Fe-deficient conditions. (A) Growth performance of WT, mpk3, and mpk6 mutant plants. 30 day-old seedlings were grown in Fe -sufficient $(50 \mu \mathrm{M})$ or -deficient $(0 \mu \mathrm{M})$ nutrient solution for 7 days. (B) Young leaves (from the third to the eighth) were detached to display their phenotypes. (C) SPAD values of WT, mpk3, and mpk6 mutants. (D) Soluble Fe concentration of WT, mpk3, and mpk6 mutants. Scale bar $=3 \mathrm{~cm}$. Data are shown as the mean $\pm \operatorname{SEM}(n=3)$. Column marked with "*" indicate a significant difference $(P<0.05)$.
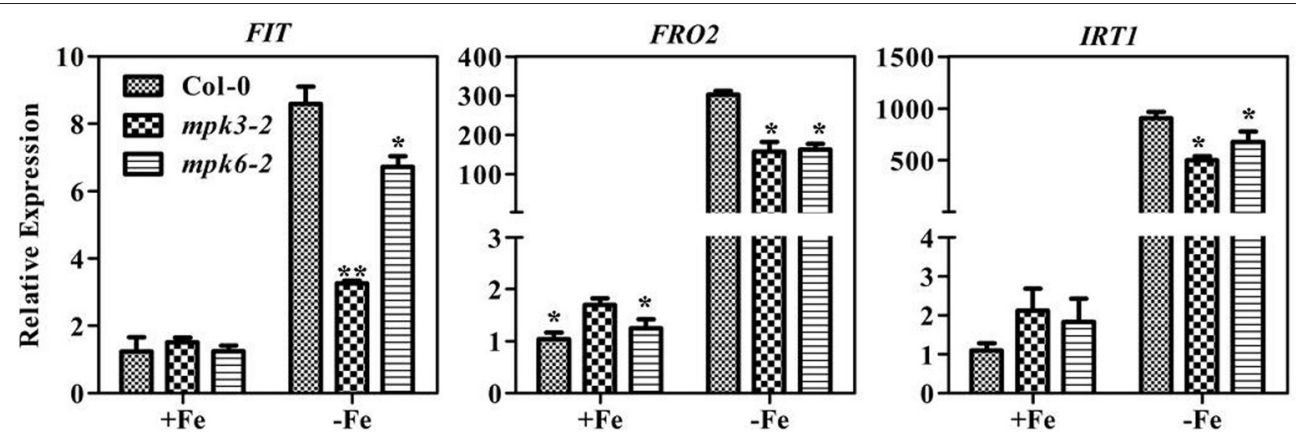

FIGURE 8 | Transcription levels of Fe-deficiency responsive genes in $\mathbf{m p k} \mathbf{3}$ and $\boldsymbol{m} \boldsymbol{p} \mathbf{k} \mathbf{6}$ mutants. Ten day old seedlings cultured in swimming medium were transferred to Fe-deficient medium for 7 days and sampled for RNA extraction. All values are expressed relative to the expression level under Fe-sufficient conditions (control-set to 1.0) as appropriate. Data are shown as the mean \pm SEM $(n=3)$. Columns marked with "*" indicate a significant difference $(P<0.05)$, and "**" indicate a highly significantly difference $(P<0.01)$.

ACS11 in this study was not detected by García et al. (2010). The inconsistency between the two studies may be attributed to the different experimental conditions used. While García et al. (2010) examined the expression of ACS genes at $24 \mathrm{~h}$ after -Fe treatment, we did qRT-PCR on the plant tissues that had been treated with Fe deficiency for 7 days. Seven days of Fe deprivation was chosen as that is when chlorotic symptoms are visible (Supplementary Figure 5). According to Vert et al. (2003), the level of IRT1 and $\mathrm{FRO} 2$ transcripts increases at $3 \mathrm{~d}$ and reaches to the maximum level at $5 \mathrm{~d}$ after $-\mathrm{Fe}$ treatment. In their paper, the IRT1 and FRO2 transcript abundance at day 7 is similar to day 3 of Fe deficiency (Vert et al., 2003). To verify that, we did a time-course qRTPCR analysis using plant samples with different period of -Fe treatment, including 1, 3, 5, and 7 days of Fe-deprived treatment. Results showed that the up-regulation levels of the tested -Fe induced genes were stable from day 1 to day 7 of the treatment (Supplementary Figure 6). Thus, the expression of Fe acquisition related genes in response to 7 days of Fe deficiency is a good reflection of the transcript abundance for the genes measured. As to the expression of the ACS genes, after 7 days of -Fe treatment, 
the up-regulation of rapid response $A C S$ genes may have returned to a normal level. On the other hand, the long term Fe deficiency may turn on the general stress responsive genes, which is not directly related to Fe deficiency. Whether ACS4 and ACS11 are response to short or long term Fe-deficiency differently needs to be further investigated.

In summary, we demonstrated that the MPK3/MPK6 participates in Fe deficiency-induced ethylene production. Loss function in MPK3 and MPK6, or their downstream ACS2 isoform suppressed the Fe deficient responses. Roles of the other Fe-responsive ACS isoforms, such as ACS7, ACS 9, and ACS11 at leaves and roots, and ACS8 in leaves, in Fe deficiency-induced ethylene production remained to be explored. Additional studies, including studies of the regulation of upstream genes of MPK3/MPK6 in Fe deficiency-induced ethylene production and of other ACS isoforms would expand our understanding of

\section{REFERENCES}

Chae, H. S., and Kieber, J. J. (2005). Eto Brute? Role of ACS turnover in regulating ethylene biosynthesis. Trends Plant Sci. 10, 291-296. doi: 10.1016/j.tplants.2005.04.006

Conte, S. S., and Walker, E. L. (2011). Transporters contributing to iron trafficking in plants. Mol. Plant 4, 464-476. doi: 10.1093/mp/ssr015

Curie, C., Cassin, G., Couch, D., Divol, F., Higuchi, K., Le Jean, M., et al. (2009). Metal movement within the plant: contribution of nicotianamine and yellow stripe 1-like transporters. Ann. Bot. Lond. 103, 1-11. doi: 10.1093/aob/mcn207

Curie, C., Panaviene, Z., Loulergue, C., Dellaporta, S. L., Briat, J.-F., and Walker, E. L. (2001). Maize yellow stripe1 encodes a membrane protein directly involved in Fe(III) uptake. Nature 409, 346-349. doi: 10.1038/35053080

Eide, D., Broderius, M., Fett, J., and Guerinot, M. L. (1996). A novel iron-regulated metal transporter from plants identified by functional expression in yeast. Proc. Natl. Acad. Sci. U.S.A. 93, 5624-5628. doi: 10.1073/pnas.93.11.5624

García, M. J., Lucena, C., Romera, F. J., Alcántara, E., and Pérez-Vicente, R. (2010). Ethylene and nitric oxide involvement in the up-regulation of key genes related to iron acquisition and homeostasis in Arabidopsis. J. Exp. Bot. 61, 3885-3899. doi: $10.1093 /$ jxb/erq203

Group, M., Ichimura, K., Shinozaki, K., Tena, G., Sheen, J., Henry, Y., et al. (2002). Mitogen-activated protein kinase cascades in plants: a new nomenclature. Trends Plant Sci. 7, 301-308. doi: 10.1016/S1360-1385(02)02302-6

Han, L., Li, G.-J., Yang, K.-Y., Mao, G., Wang, R., Liu, Y., et al. (2010). Mitogen-activated protein kinase 3 and 6 regulate Botrytis cinerea-induced ethylene production in Arabidopsis. Plant J. 64, 114-127. doi: 10.1111/j.1365313x.2010.04318.x

Ivanov, R., Brumbarova, T., and Bauer, P. (2012). Fitting into the harsh reality: regulation of iron-deficiency responses in dicotyledonous plants. Mol. Plant 5, 27-42. doi: 10.1093/mp/ssr065

Kamiyoshihara, Y., Iwata, M., Fukaya, T., Tatsuki, M., and Mori, H. (2010). Turnover of LeACS2, a wound-inducible 1-aminocyclopropane-1-carboxylic acid synthase in tomato, is regulated by phosphorylation/dephosphorylation. Plant J. 64, 140-150. doi: 10.1111/j.1365-313x.2010.04316.x

Kim, C. Y., Liu, Y., Thorne, E. T., Yang, H., Fukushige, H., Gassmann, W., et al. (2003). Activation of a stress-responsive mitogen-activated protein kinase cascade induces the biosynthesis of ethylene in plants. Plant Cell 15, 2707-2718. doi: $10.1105 /$ tpc. 011411

Kim, S. A., and Guerinot, M. L. (2007). Mining iron: iron uptake and transport in plants. FEBS Lett. 581, 2273-2280. doi: 10.1016/j.febslet.2007.04.043

Kobayashi, T., and Nishizawa, N. K. (2012). Iron uptake, translocation, and regulation in higher plants. Annu. Rev. Plant Biol. 63, 131-152. doi: 10.1146/annurev-arplant-042811-105522

Lee, J. S., and Ellis, B. E. (2007). Arabidopsis MAPK phosphatase 2 (MKP2) positively regulates oxidative stress tolerance and inactivates the MPK3 and MPK6 MAPKs. J. Biol. Chem. 282, 25020-25029. doi: 10.1074/jbc.M701888200 the regulatory mechanisms of ethylene induction under $\mathrm{Fe}$ deficiency.

\section{ACKNOWLEDGMENTS}

This work was supported by the National Natural Science Foundation $(31172024,31401934)$ and the 111 project (B14027). The acs mutants were obtained from the Arabidopsis Biological Resource Center (ABRC). The authors are grateful to Dr. Chuang Wang for critical reading of this manuscript.

\section{SUPPLEMENTARY MATERIAL}

The Supplementary Material for this article can be found online at: http://journal.frontiersin.org/article/10.3389/fpls.2015. 00953

Lei, L., Li, Y., Wang, Q., Xu, J., Chen, Y., Yang, H., et al. (2014). Activation of MKK9-MPK3/MPK6 enhances phosphate acquisition in Arabidopsis thaliana. New Phytol. 203, 1146-1160. doi: 10.1111/nph.12872

Li, G., Meng, X., Wang, R., Mao, G., Han, L., Liu, Y., et al. (2012). Duallevel regulation of ACC synthase activity by MPK3/MPK6 cascade and its downstream WRKY transcription factor during ethylene induction in Arabidopsis. PLoS Genet. 8:e1002767. doi: 10.1371/journal.pgen. 1002767

$\mathrm{Li}, \mathrm{X}$., and Li, C. (2004). Is ethylene involved in regulation of root ferric reductase activity of dicotyledonous species under iron deficiency? Plant Soil 261, 147-153. doi: 10.1023/B:PLSO.0000035536.79001.60

Lingam, S., Mohrbacher, J., Brumbarova, T., Potuschak, T., Fink-Straube, C., Blondet, E., et al. (2011). Interaction between the bHLH transcription factor FIT and ETHYLENE INSENSITIVE3/ETHYLENE INSENSITIVE3LIKE1 reveals molecular linkage between the regulation of iron acquisition and ethylene signaling in Arabidopsis. Plant Cell 23, 1815-1829. doi: 10.1105/tpc.111.084715

Liu, H., Wang, Y., Xu, J., Su, T., Liu, G., and Ren, D. (2007). Ethylene signaling is required for the acceleration of cell death induced by the activation of AtMEK5 in Arabidopsis. Cell Res. 18, 422-432. doi: 10.1038/cr.2008.29

Liu, Y., and Zhang, S. (2004). Phosphorylation of 1-aminocyclopropane-1carboxylic acid synthase by MPK6, a stress-responsive mitogen-activated protein kinase, induces ethylene biosynthesis in Arabidopsis. Plant Cell 16, 3386-3399. doi: 10.1105/tpc.104.026609

Lucena, C., Waters, B. M., Romera, F. J., García, M. J., Morales, M., Alcántara, E., et al. (2006). Ethylene could influence ferric reductase, iron transporter, and $\mathrm{H}+$-ATPase gene expression by affecting FER (or FER-like) gene activity. J. Exp. Bot. 57, 4145-4154. doi: 10.1093/jxb/erl189

Pedley, K. F., and Martin, G. B. (2005). Role of mitogen-activated protein kinases in plant immunity. Curr. Opin. Plant Biol. 8, 541-547. doi: 10.1016/j.pbi.2005.07.006

Pitzschke, A., Schikora, A., and Hirt, H. (2009). MAPK cascade signalling networks in plant defence. Curr. Opin. Plant Biol. 12, 421-426. doi: 10.1016/j.pbi.2009.06.008

Ren, D., Yang, H., and Zhang, S. (2002). Cell death mediated by MAPK is associated with hydrogen peroxide production in Arabidopsis. J. Biol. Chem. 277, 559-565. doi: 10.1074/jbc.M109495200

Robinson, N. J., Procter, C. M., Connolly, E. L., and Guerinot, M. L. (1999). A ferric-chelate reductase for iron uptake from soils. Nature 397, 694-697. doi: $10.1038 / 17800$

Rodriguez, M. C. S., Petersen, M., and Mundy, J. (2010). Mitogen-activated protein kinase signaling in plants. Annu. Rev. Plant Biol. 61, 621-649. doi: 10.1146/annurev-arplant-042809-112252

Romera, F. J., and Alcantara, E. (1994). Iron-deficiency stress responses in cucumber (Cucumis sativus L.) roots (a possible role for ethylene?). Plant Physiol. 105, 1133-1138. 
Romera, F. J., and Alcantara, E. (2004). Ethylene involvement in the regulation of Fe-deficiency stress responses by Strategy I plants. Funct. Plant Biol. 31, 315-328. doi: 10.1071/FP03165

Romera, F. J., Alcantara, E., and De La Guardia, M. D. (1999). Ethylene production by fe-deficient roots and its involvement in the regulation of fedeficiency stress responses by strategy I plants. Ann. Bot.Lond. 83, 51-55. doi: 10.1006/anbo.1998.0793

Romera, F. J., García, M. J., Alcántara, E., and Pérez-Vicente, R. (2011). Latest findings about the interplay of auxin, ethylene and nitric oxide in the regulation of Fe deficiency responses by Strategy I plants. Plant Signal. Behav. 6, 167-170. doi: $10.4161 /$ psb.6.1.14111

Santi, S., and Schmidt, W. (2009). Dissecting iron deficiency-induced proton extrusion in Arabidopsis roots. New Phytol. 183, 1072-1084. doi: 10.1111/j.1469-8137.2009.02908.x

Sato, T., and Theologis, A. (1989). Cloning the mRNA encoding 1aminocyclopropane-1-carboxylate synthase, the key enzyme for ethylene biosynthesis in plants. Proc. Natl. Acad. Sci. U.S.A. 86, 6621-6625. doi: 10.1073/pnas.86.17.6621

Takahashi, F., Yoshida, R., Ichimura, K., Mizoguchi, T., Seo, S., Yonezawa, M., et al. (2007). The mitogen-activated protein kinase cascade MKK3-MPK6 is an important part of the jasmonate signal transduction pathway in Arabidopsis. Plant Cell 19, 805-818. doi: 10.1105/tpc.106.046581

Teige, M., Scheikl, E., Eulgem, T., Dóczi, R., Ichimura, K., Shinozaki, K., et al. (2004). The MKK2 pathway mediates cold and salt stress signaling in Arabidopsis.Mol. Cell 15, 141-152. doi: 10.1016/j.molcel.2004.06.023

Tena, G., Boudsocq, M., and Sheen, J. (2011). Protein kinase signaling networks in plant innate immunity. Curr. Opin. Plant Biol. 14, 519-529. doi: 10.1016/j.pbi.2011.05.006

Tsuchisaka, A., Yu, G., Jin, H., Alonso, J. M., Ecker, J. R., Zhang, X., et al. (2009). A combinatorial interplay among the 1-aminocyclopropane-1-carboxylate isoforms regulates ethylene biosynthesis in Arabidopsis thaliana. Genetics 183, 979-1003. doi: 10.1534/genetics.109.107102

Tsuda, K., Sato, M., Stoddard, T., Glazebrook, J., and Katagiri, F. (2009). Network properties of robust immunity in plants. PLoS Genet. 5:e10007. doi: 10.1371/journal.pgen.1000772

Vert, G. A., Briat, J. F., and Curie, C. (2003). Dual regulation of the Arabidopsis high-affinity root iron uptake system local and long-distance signals. Plant Physiol. 132, 796-804. doi: 10.1104/pp.102.016089

Walker, E. L., and Connolly, E. L. (2008). Time to pump iron: iron-deficiencysignaling mechanisms of higher plants. Curr. Opin. Plant Biol. 11, 530-535. doi: 10.1016/j.pbi.2008.06.013

Wang, H., Liu, Y., Bruffett, K., Lee, J., Hause, G., Walker, J. C., et al. (2008). Haploinsufficiency of MPK3 in MPK6 mutant background uncovers a novel function of these two MAPKs in Arabidopsis ovule development. Plant Cell 20, 602-613. doi: $10.1105 /$ tpc. 108.058032

Wang, H., Ngwenyama, N., Liu, Y., Walker, J. C., and Zhang, S. (2007). Stomatal development and patterning are regulated by environmentally responsive mitogen-activated protein kinases in Arabidopsis. Plant Cell 19, 63-73. doi: 10.1105/tpc.106.048298

Wang, K. L.-C., Li, H., and Ecker, J. R. (2002). Ethylene biosynthesis and signaling networks. Plant Cell 14, 131-151. doi: 10.1105/tpc.001768

Wang, P., Du, Y., Li, Y., Ren, D., and Song, C. P. (2010). Hydrogen peroxidemediated activation of MAP kinase 6 modulates nitric oxide biosynthesis and signal transduction in Arabidopsis. Plant Cell 22, 2981-2998. doi: 10.1105/tpc.109.072959

Waters, B. M., Lucena, C., Romera, F. J., Jester, G. G., Wynn, A. N., Rojas, C. L., et al. (2007). Ethylene involvement in the regulation of the H+-ATPase CsHA1 gene and of the new isolated ferric reductase CsFRO1 and iron transporter CsIRT1 genes in cucumber plants. Plant Physiol. Biochem. 45, 293-301. doi: 10.1016/j.plaphy.2007.03.011

Xu, J., Li, Y., Wang, Y., Liu, H., Lei, L., Yang, H., et al. (2008). Activation of MAPK kinase 9 induces ethylene and camalexin biosynthesis and enhances sensitivity to salt stress in Arabidopsis. J. Biol. Chem. 283, 26996-27006. doi: 10.1074/jbc.M801392200

Yang, S. F., and Hoffman, N. E. (1984). Ethylene biosynthesis and its regulation in higher plants. Ann. Rev. Plant. Physiol. 35, 155-189. doi: 10.1146/annurev.pp.35.060184.001103

Yoo, S.-D., Cho, Y.-H., Tena, G., Xiong, Y., and Sheen, J. (2008). Dual control of nuclear EIN3 by bifurcate MAPK cascades in C2H4 signalling. Nature 451, 789-795. doi: 10.1038/nature06543

Zarembinski, T., and Theologis, A. (1994). "Ethylene biosynthesis and action: a case of conservation," in Signals and Signal Transduction Pathways in Plants, ed K. Palme (Albany, NY: Springer), 343-361.

Zhang, J., Shao, F., Li, Y., Cui, H., Chen, L., Li, H., et al. (2007). A Pseudomonas syringae effector inactivates MAPKs to suppress PAMP-induced immunity in plants. Cell Host Microbe 1, 175-185. doi: 10.1016/j.chom.2007.03.006

Zheng, L., Huang, F., Narsai, R., Wu, J., Giraud, E., He, F., et al. (2009). Physiological and transcriptome analysis of iron and phosphorus interaction in rice seedlings. Plant Physiol. 151, 262-274. doi: 10.1104/pp.109.141051

Zhou, C., Cai, Z., Guo, Y., and Gan, S. (2009). An Arabidopsis mitogen-activated protein kinase cascade, MKK9-MPK6, plays a role in leaf senescence. Plant Physiol. 150, 167-177. doi: 10.1104/pp.108.133439

Conflict of Interest Statement: The authors declare that the research was conducted in the absence of any commercial or financial relationships that could be construed as a potential conflict of interest.

Copyright (c) 2015 Ye, Li, Wang, Wang, Li, Du, Zhang and Shou. This is an openaccess article distributed under the terms of the Creative Commons Attribution License (CC BY). The use, distribution or reproduction in other forums is permitted, provided the original author(s) or licensor are credited and that the original publication in this journal is cited, in accordance with accepted academic practice. No use, distribution or reproduction is permitted which does not comply with these terms. 